\title{
OPTIMIZATION OF NATURAL GAS TRANSPORT PIPELINE NETWORK LAYOUT: A NEW METHODOLOGY BASED ON DOMINANCE DEGREE MODEL
}

\author{
Zhenjun Zhu' ${ }^{1}$, Chaoxu Sun ${ }^{2}$, Jun Zeng ${ }^{1}$, Guowei Chen ${ }^{3}$ \\ ${ }^{1}$ Jiangsu Key Laboratory of Urban ITS, School of Transportation, Southeast University, Nanjing, China \\ ${ }^{2}$ Zhejiang Provincial Natural Gas Development Co. Ltd, Hangzhou, China \\ ${ }^{3}$ Zhejiang Electric power construction Co. Ltd, Ningbo, China
}

Submitted 23 January 2017; resubmitted 13 April 2017; accepted 20 April 2017

\begin{abstract}
At the phase of 13-th five-year plan in China, natural gas will play an important role in energy revolution. With the growth of consumption, natural gas infrastructures will become hot spots of future investment and pipeline network construction will also usher in a period of rapid development. Therefore, it is of great theoretical and practical significance to study layout methods of transport pipeline network. This paper takes natural gas transport pipeline network as a research object, introduces dominance degree to analyse benefits of pipeline projects. Then, this paper proposes Dominance Degree Model (DDM) of transport pipeline projects based on Potential Model (PM) and Economic Potential Theory (EPT). According to DDM of gas transport pipeline projects, layout methods of pipeline network are put forward, which is simple and easy to obtain the overall optimal solution and ensure maximum comprehensive benefits. What's more, construction sequences of gas transport pipeline projects can be also determined. Finally, the model is applied to a real case of natural gas transport pipeline projects in Zhejiang Province, China. The calculation results suggest that the model should deal with the transport pipeline network layout problem well, which have important implications for other potential pipeline networks not only in the Zhejiang Province but also throughout China and beyond.
\end{abstract}

Keywords: natural gas; transport pipeline network; dominance degree model; potential model; economic potential theory; layout method.

\section{Introduction}

As an efficient and cleaner energy, natural gas is an important bridge to make energy consumption transit to low-carbon (Gillingham et al. 2009). According to 13th five-year plan and National Energy Development Strategy Plan of China, with respect to the proportion of primary energy consumption, natural gas demand will continue to increase, its share will reach $10 \%$ by the year of 2020. Continued growth in consumption is bound to promote the construction of natural gas infrastructures, coverage area of trunk pipelines will in further expansion. Thus, gas transport and distribution network will be improved and facilities in different pipe network will also achieve interoperability gradually.

Natural gas transport pipeline network shoulders the task of gas supply, which plays a significant role in improving the overall socio-economic benefits for the region. However, pipeline projects' investment is large and payback period is long, which cannot be easily re- constructed or expanded. So when we make the pipeline network layout, market supply and demand, benefits and construction costs of pipeline projects' investments, etc. - should be considered to determine a reasonable layout scheme.

A number of studies have been conducted on layout optimization with respect to natural gas transport pipeline network, where most of this research has focused on mathematical optimization. For example, Edgar et al. (1978) used Generalized Reduced Gradient Method to optimize natural gas transport pipeline network system. Bhaskaran and Salzborn (1979) studied the optimal design problem of gas gathering pipeline network, which was divided into three sub-problem: system layout, node locations, and the diameter distribution. Pedrycz et al. (1992) examined the design and application of neural networks in the context of a specific decision-making problem, the selection of an appropri-

Corresponding author: Zhenjun Zhu

E-mail: zhuzhenjun@seu.edu.cn 
ate layout for natural gas distribution system. Singh and Nain (2012), Sanaye and Mahmoudimehr (2013) applied genetic algorithm to pipeline system optimization and simulated natural gas transport pipeline network. Üster and Dilaveroğlu (2014) developed an integrated largescale mixed-integer nonlinear optimization model to determine pipelines in the network. Schmidt et al. (2015) presented stationary neuro-linguistic programming type models of gas networks that are primarily designed to include detailed nonlinear physics in the final optimization steps for mid-term planning problems. An and Peng (2016) used risk cost functions to study the synchronization of the minimum risk loss and total cost of natural gas pipeline networks at the planning stage. However, these methods are essentially solving network structure to satisfy a given criterion, a local optimal solution can be only obtained.

Minimum Spanning Tree Method (MSTM) and Dynamic Programming (DP) are the most commonly used solutions. MSTM abstracts pipeline network into undirected network, including classic solutions of Dijkstra Algorithms, Kruskal Algorithms, Steiner Algorithms (Graham, Hell 1985). Compared to solutions of traditional graph theory, these algorithms adopt computer programs for processing, whose operation efficiency is relatively high, Steiner Algorithms effectively solve to the shortest path problem of large-scale network (Han, Lim 2010; Sniedovich 2006; Zhou 2004). However, the above three algorithms do not consider investment costs of pipeline projects, whose results can be only regarded as the initial pipeline network layout. DP can deal the optimization problem of multiple decision-making variables, while 'dimension obstacles' exist in solving, that is computation will increase exponentially with growth of the number of variables. When the dimension of this problem increases to a certain degree, it cannot be solved (Bellman, Dreyfus 2016). Through the above analysis, we can see that current commonly used methods have certain advantages and limitations, as shown in Table 1.

In summary, layout optimization of regional natural gas transmission pipeline network is a multi-objec- tive nonlinear programming problem; the above methods are difficult to solve the problem effectively in the practical application. Compared to the existing methods, a new methodology considering practical application is proposed to simplify computing and obtain the overall optimal solution in this paper, which can not only determine the optimal layout scheme, but also obtain construction sequences of gas transport pipeline projects, which ensure maximum comprehensive benefits of pipeline network. Specifically, we combine Potential Model (PM) and Economic Potential Theory (EPT) to establish the Dominance Degree Model (DDM) for pipeline projects, and use dominance degree to measure comprehensive benefits of pipeline projects. Then, a layout method based on the DDM for natural gas transport pipeline network is proposed, which can search out the most advantageous pipeline project successively through cycle calculations. The calculation process is simple and easy to obtain the overall optimal solution. To verify validity of the method, natural gas transport pipeline projects in Zhejiang Province are taken as a case study.

\section{Dominance Degree of Pipeline Projects}

Dominance generally refers to the advantageous form that one party can overcome or overwhelm its counterpart. Dominance degree is an index, which can be used to reflect and compare the degree of the pros and cons among system elements (Shamsie 2003). Due to differences in location, economy and geographical conditions, transport pipeline projects have made a tremendous impact on social and economic benefits and influenced dominance degree of network layout. Therefore, this paper uses dominance degree to reflect comprehensive benefits of transport pipeline projects and compare their comparative dominance.

As for regional natural gas transport pipeline network, dominance degree of transport pipeline projects are closely related to influential factors, such as functional orientation, scale, economic development level of cities and towns, construction costs of transport pipeline projects, etc.

Table 1. The methodology analysis

\begin{tabular}{|c|c|c|}
\hline Methods & Advantages & Limitations \\
\hline Graph theory & $\begin{array}{l}\text { - easy access to computer program processing; } \\
\text { - higher computing efficiency; } \\
\text { - solving the shortest tree problem within a large } \\
\text { scale network effectively }\end{array}$ & $\begin{array}{l}\text { - only solving the network layout among known fixed } \\
\text { points; } \\
\text { - project investment costs are not considered; } \\
\text { - solutions can only be used as the initial network layout }\end{array}$ \\
\hline $\begin{array}{l}\text { Dynamic } \\
\text { programming }\end{array}$ & $\begin{array}{l}\text { - solving optimization problems with multiple } \\
\text { decision-making variables }\end{array}$ & $\begin{array}{l}\text { - not suitable for dealing with large-scale network systems, } \\
\text { 'dimension obstacles' exist in solving }\end{array}$ \\
\hline $\begin{array}{l}\text { Neural network } \\
\text { method }\end{array}$ & $\begin{array}{l}\text { - solving optimization problems with multiple } \\
\text { decision-making variables }\end{array}$ & - only obtaining the local optimal solution \\
\hline $\begin{array}{l}\text { Genetic } \\
\text { algorithm }\end{array}$ & $\begin{array}{l}\text { - higher adaptability, which can overcome the dif- } \\
\text { ficulties in solving nonlinear optimization }\end{array}$ & $\begin{array}{l}\text { - lower computing efficiency; } \\
\text { - no effective quantitative analysis concerning algorithm } \\
\text { precision, feasibility and computational complexity }\end{array}$ \\
\hline $\begin{array}{l}\text { Complex } \\
\text { method }\end{array}$ & $\begin{array}{l}\text { - the algorithm is simple and suitable for dealing } \\
\text { with constrained optimization problems }\end{array}$ & $\begin{array}{l}\text { - unable to deal with multi-variable, multi-constraint op- } \\
\text { timization problems }\end{array}$ \\
\hline
\end{tabular}




\subsection{Functional Orientation of Cities and Towns}

Functional orientation of cities and towns is the core of their development and competition, cities and towns that play a dominant role in socio-economic development are important strategic nodes of regional natural gas transport pipeline network layout. They have different development advantages, such as location, resource and policy, etc. They can also maximize the optimal resource configuration, which offers good conditions for expanding natural gas consumption market.

\subsection{Scale and Economic Development Level of Cities and Towns}

Natural gas consumption is closely related to scale and economic development level of cities and towns, which determine consumption capacity and growth speed of natural gas. In order to make natural gas consumption market have more potential, transport pipeline projects require cities and towns with a certain concentration of consumer groups and economic strength. Therefore, scale and economic development level are the main factors that affect natural gas consumption.

\subsection{Construction Costs of Pipeline Projects}

Construction costs of pipeline projects are related to its length and costs of crossing obstacles, which directly affect the investment and benefits of projects. Natural gas transport pipeline projects have a certain service life, the more construction costs we input, the longer payback period and the weaker profitability it will be. Therefore, construction costs should be considered to ensure profitability when making natural gas transport pipeline network layout.

\section{Related Theories and Models}

\subsection{Potential Model}

PM is a commonly used model in the empirical study of regional economy, which is mainly used to measure a point influenced by a set of given points in the space. Firstly, interaction of a node with each other node is calculated. Then, comprehensive influence is obtained by summing up interaction of a node with each other point (Mátyás 1998; Lu, Taur 2006). Total potential of node $i$ can be expressed as follows:

$$
V_{i}=K \cdot \sum_{j} \frac{m_{j}}{d_{i j}},
$$

where: $K$ is the dielectric constant; $m_{j}$ is the scale of point $j$, according to different research questions, population and economy scale can be selected; $d_{i j}$ is the impedance between $i$ and $j$.

In terms of cities and towns within the study area, PM can be used to analyse potential social and economic benefits generated by transport pipeline projects and compare their advantages and disadvantages. Therefore, this paper introduces PM to analyse dominance degree of natural gas transport pipeline projects.

\subsection{Economic Potential Theory}

EPT is a manifestation trend of economic energy (Chang 2008). Natural gas transport pipelines undertake the important task of transporting natural gas, which plays a significant role in optimizing regional energy consumption structure, promoting economic development and improving environment quality. There, we consider natural gas transport pipelines will generate strong economic potential in its gas supply region.

According to the electromagnetic theory, currentcarrying conductors will generate a magnetic field in its surroundings. Thus, each point in vicinity of current-carrying conductors owns the potential condition (Novoselov et al. 2004). Pipeline projects are just like current-carrying conductors, the impact on cities and towns is related to gas transport flow, the distance between cities and off-take stations, development level. In this paper, pipeline projects are simulated into currentcarrying conductors, gas transport flow is simulated into electric current, the city $P$ and off-take station $A$ are simulated into nodes. Field intensity can be used to describe economic potential of node $P$, which can be repressed as follows:

$$
B=\frac{\mu \cdot I}{a},
$$

where: $\mu$ is undetermined coefficient; $I$ is natural gas transport flow from node $A$ to $P$; $a$ is the distance between node $P$ (cities or towns) and node $A$ (off-take stations).

\section{Methodology}

Comprehensive benefits of pipeline projects are related to functional orientation, scale, economic development level of cities or towns. Through the analysis of these influential factors, we find that PM can be used to analyse the dominance degree of pipeline projects. The specific construction method is shown in Fig. 1.

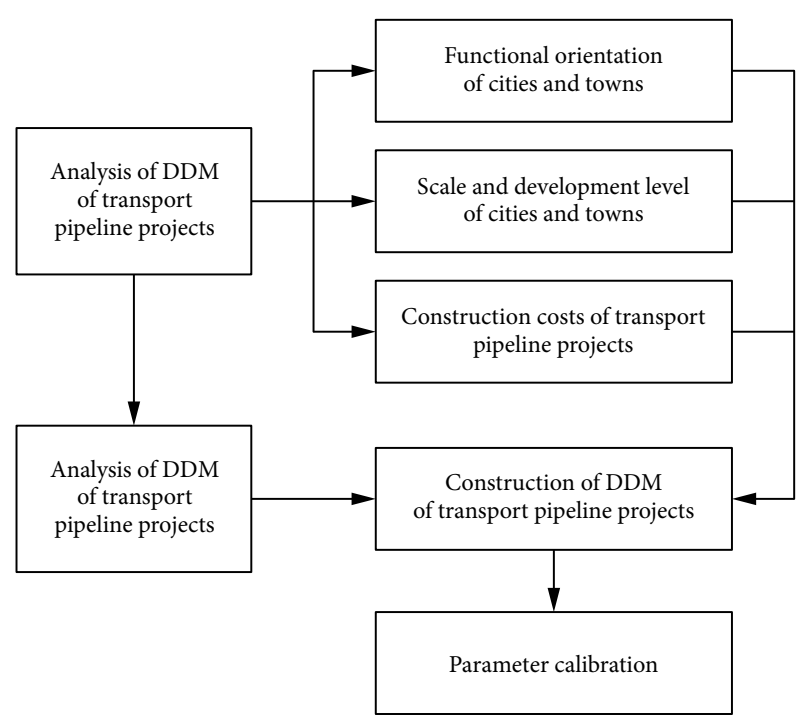

Fig. 1. Flow chart of model construction 


\subsection{Model Construction}

Based on PM and EPT, this paper proposes following assumptions and methods when constructing DDM:

- pipeline projects will generate economic potential, which can be regarded as an index reflecting city scale;

- construction costs of pipeline projects will affect their economical benefits directly, therefore, construction costs are taken as parameter reflecting impedance in PM;

- the city's economic potential influenced by pipeline projects is closely related to its development level, pipeline projects will directly affect the transformation of economic potential.

Therefore, some specific indicators can be selected to reflect these influential factors. Above all, the DDM of gas transport pipeline projects is established as follows:

$$
A_{i j}=K_{i} \cdot \frac{\left(\mu_{i} \cdot I_{i}\right) / a_{i j}}{f_{i j}},
$$

where: $A_{i j}$ is the dominance degree of pipeline projects between city $i$ and gas transport station $j ; K_{i}$ is the dielectric constant, which is a constant within a specific period for specific cities; $\mu_{i}$ is the sensitivity coefficient and reflects sensitive degree that cities put on pipeline projects; $I_{i}$ is the gas demand of city $I ; a_{i j}$ is the distance between city $i$ and gas transport station $j$, which can be expressed by straight-line distance; $f_{i j}$ is construction costs of pipeline projects between city $i$ and natural gas transport station $j$.

\subsection{Parameter Calibration}

Parameter calibration is critical for model application, this paper proposes following calibration methods for model parameters.

$K_{i}$ is the dielectric constant, As for DDM of natural gas transport pipeline projects, which is closely related to urban functional orientation of cities and towns. Functional orientation is a manifestation of government functions, which guides many planning system, such as urban policies, industry structure, etc. City scale is of great significance for functional orientation and relatively stable in a long period of time. Therefore, in order to simplify this question, urban scale $P$ is selected to reflect this parameter. With the reference to notification about adjusting classification criteria for city scale of 2014, $K_{i}$ can be calibrated as shown in Table 2 .

$\mu_{i}$ is related to the richness of urban resources, market development level, living standards of residents, the existing state of industry. In this paper, Gross Domestic Product (GDP) per capital of macroeconomic indicators is used to represent this parameter.

$I_{i}$ is the gas demand of city $i$, which is related to local energy consumption structure and policies. In order to facilitate analysis of this question, energy consumption per unit GDP is selected to reflect energy consumption and saving status. To some extent, it reflects current gas demand and development potential of future natural gas. $f_{i j}$ is construction costs of pipeline projects between city $i$ and gas transport station $j$, which will directly affect investment income. Natural gas transport pipeline projects will cross rivers, roads, railways, etc. Thus, construction costs are mainly determined by pipeline length and investment for crossing obstacles. Therefore, the calculation method of $f_{i j}$ is shown as follows:

$$
f_{i j}=V \cdot G \cdot \beta \cdot a_{i j}+\sum_{k=1}^{4} m_{k} \cdot w_{k},
$$

where: $V$ is pipeline price per unit weight; $G$ is pipeline weight per unit length (the value of $V$ and $G$ can take pipelines of the same diameter, material, thickness as standard); $\beta$ is terrain correction coefficient, which is used to modify pipeline route length (different topography conditions are endowed with different values, which can be determined by referring to engineering data within the study area); $m_{k}$ is crossing times of the $k$-th type' $w_{k}$ is the corresponding construction costs $(k=1$, $2,3,4$ respectively represents the river, grade highway, substandard highway and railway).

Table 2. Calibration for the value of $K_{i}$

\begin{tabular}{|c|c|}
\hline$P[10000$ people $]$ & $K_{i}$ \\
\hline$<20$ & 1 \\
\hline $20-50$ & 1.25 \\
\hline $50-100$ & 1.5 \\
\hline $100-300$ & 2.0 \\
\hline $300-500$ & 2.5 \\
\hline $500-1000$ & 3.0 \\
\hline
\end{tabular}

\subsection{The Layout Method Based on DDM}

DDM of pipeline projects fully embody comprehensive socio-economic benefits of the projects. Apply DDM to natural gas transport pipeline network layout will maximum socio-economic benefits. Through the analysis of DDM of pipeline projects, the optimal layout scheme and construction sequences are determined, which will give full play to advantages of pipeline projects and promote regional socio-economic development. The basic application process is shown in Fig. 2.

According to the layout process, layout methods of natural gas transport network based on DDM have four steps, which are shown as follows:

- Step 1: Abstract pipeline network in the regional area. First, abstract gas transport stations and valve chests into spatial nodes, which record a set of nodes $j=\{1,2, \ldots, m\}$. Using Geographic Information System (GIS), their locations in the map can be determined. Then, connect these nodes based on pipeline alignments and obtain spatial structure diagram of regional pipeline network;

- Step 2: Calculate the dominance degree of pipeline projects. Similarly, abstract cities and towns that uncovered by gas transmission pipeline network into a set of nodes $i=\{1,2, \ldots, n\}$. 


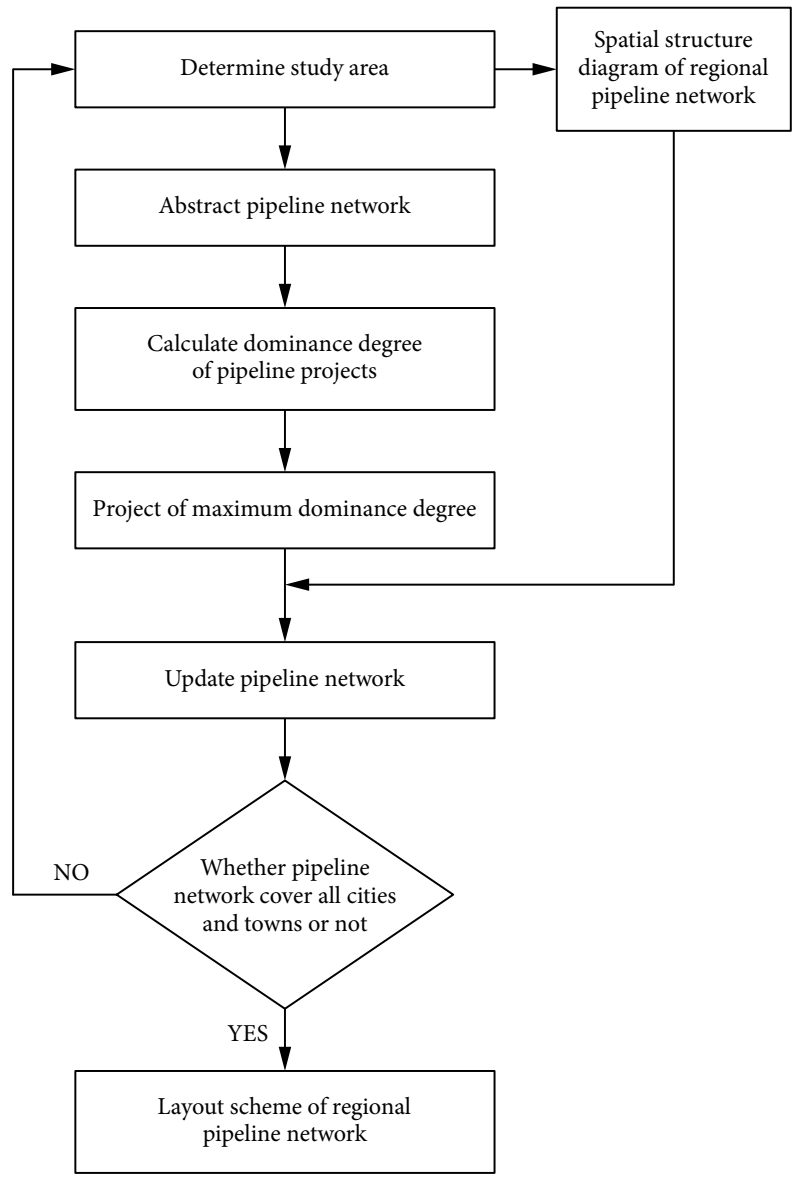

Fig. 2. Natural gas transport pipeline network layout process

Calibrate model parameters and use DDM to calculate dominance degree between node $i$ and node $j$ sequentially. Then, sort calculation results in descending order.

- Step 3: Update pipeline network in the regional area. According to calculation results, take the pipeline project of maximum dominance degree and connect its nodes at both ends to form a new pipeline route. Then, corresponding nodes $i$ is incorporated into a set of node $j$, that is $j=\{1,2$, $\ldots, m+1\}$. For example, assume that $i=\{1,2,3$, $4\}$ and $j=\{1,2\}$, the calculated maximum dominance degree $A_{21}$, which means $i=2$ is removed from a set of node $i$, subsumed into a set of node $j$ and denoted as $j=3$, then $i=\{1,3,4\}$ and $j=\{1$, $2,3\}$. Finally, updated spatial structure diagram of regional pipeline network is obtained.
- Step 4: Calculate cyclically and output the results. Repeat step 2 and step 3 until the set of nodes $i=\{1,2, \ldots, n\}$ is an empty set, while the set of nodes $j=\{1,2, \ldots, m+n\}$. At this time, all cities and towns have been covered by pipeline network and layout scheme of pipeline network is obtained.

\section{Case Study}

In this paper, Lishui region along Jinhua-Lishui-Wenzhou gas transport trunk pipeline engineering in Zhejiang Province, China is taken as a case study. DDM is used to determine the optimal layout scheme and construction sequences.

\subsection{Pipeline Network and Model Parameters}

There are three natural gas transport stations and two valve chests, GIS is used to determine their locations. Then, abstract pipeline network in the regional area as shown in Fig. 3.

According to Statistical Yearbook of Lishui City of 2015 and energy consumption statistics of each county of Lishui city (Mátyás 1998), input parameters are shown in Table 3.

Take related data of Lishui section in the JinhuaLishui-Wenzhou pipeline engineering as reference, use L450M steel for standard pipelines. The value of $\beta$ is $1.032 ; V$ is 0.77 ten thousand yuan/tons; $G$ is 222.3 tons $/ \mathrm{km} ; w_{1}, w_{2}, w_{3}, w_{4}$ are respectively $250,15,4,25$ thousand yuan/time.

\subsection{Model Calculation Results}

Take relevant parameters into the DDM, through cycle calculation, the results are obtained as shown in Table 4 .

According to calculation results, update natural gas transport pipeline network within the study area successively. Finally, the layout scheme of natural gas transport pipeline network is obtained, as shown in Fig. 4.

Based on considering economic benefits and costs, the layout scheme calculated by DDM is the overall optimal solution. Meanwhile, according to cycle calculation results, construction sequences of natural gas transport pipeline projects can be determined and maximum comprehensive benefits of natural gas transport pipeline network are ensured. Under the condition of funding constraints, the optimal construction sequence in Lishui region is arranged successively: from teminal station in Lishui City to Songyang County, Songyang County to

Table 3. Input parameters of cities and towns

\begin{tabular}{|l|c|c|c|}
\hline \multicolumn{1}{|c|}{ Name } & $K$ & $M[10000$ yuan/person] & $I$ [tons of standard coal/10000 yuan] \\
\hline Suiyang County & 1.25 & 46.596 & 0.470 \\
\hline Songyang County & 1.25 & 44.037 & 0.456 \\
\hline Yunhe County & 1 & 45.627 & 0.532 \\
\hline Jingning County & 1 & 38.541 & 0.458 \\
\hline Longquan City & 1.25 & 39.546 & 0.491 \\
\hline Qinyuan County & 1.25 & 43.943 & 0.490 \\
\hline
\end{tabular}


Suichang County, Songyang County to Yunhe County, Yunhe County to Jingning County, Yunhe County to Longquan City, Longquan City to Qinyuan County. Gas transport stations can be also obtained, which is arranged at Songyang County, Yunhe County and Longquan City.

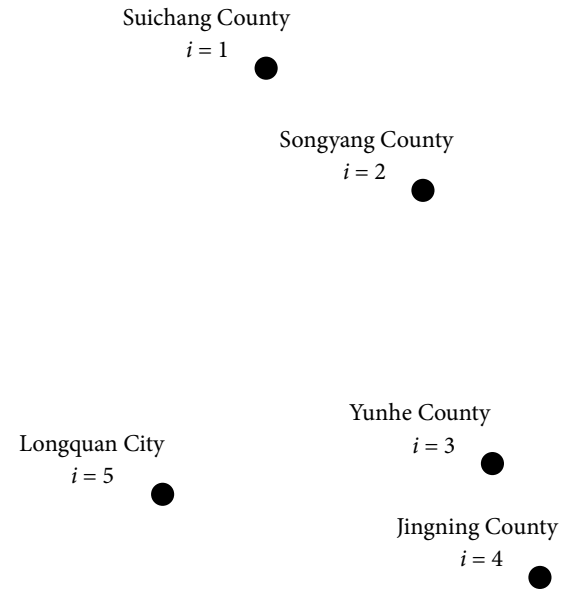

Qinyuan City

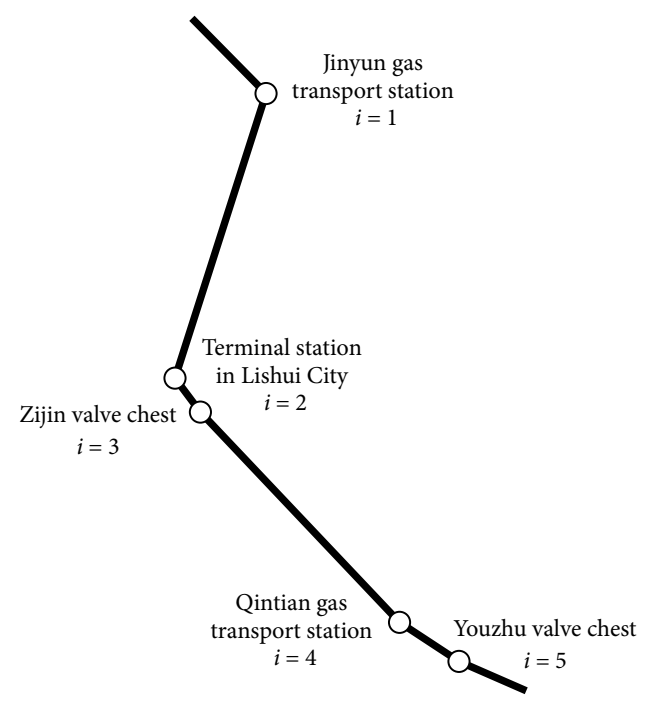

Built pipeline projects

New pipeline projects

- Cities or towns $i=6$

Fig. 3. Natural gas transport pipeline network

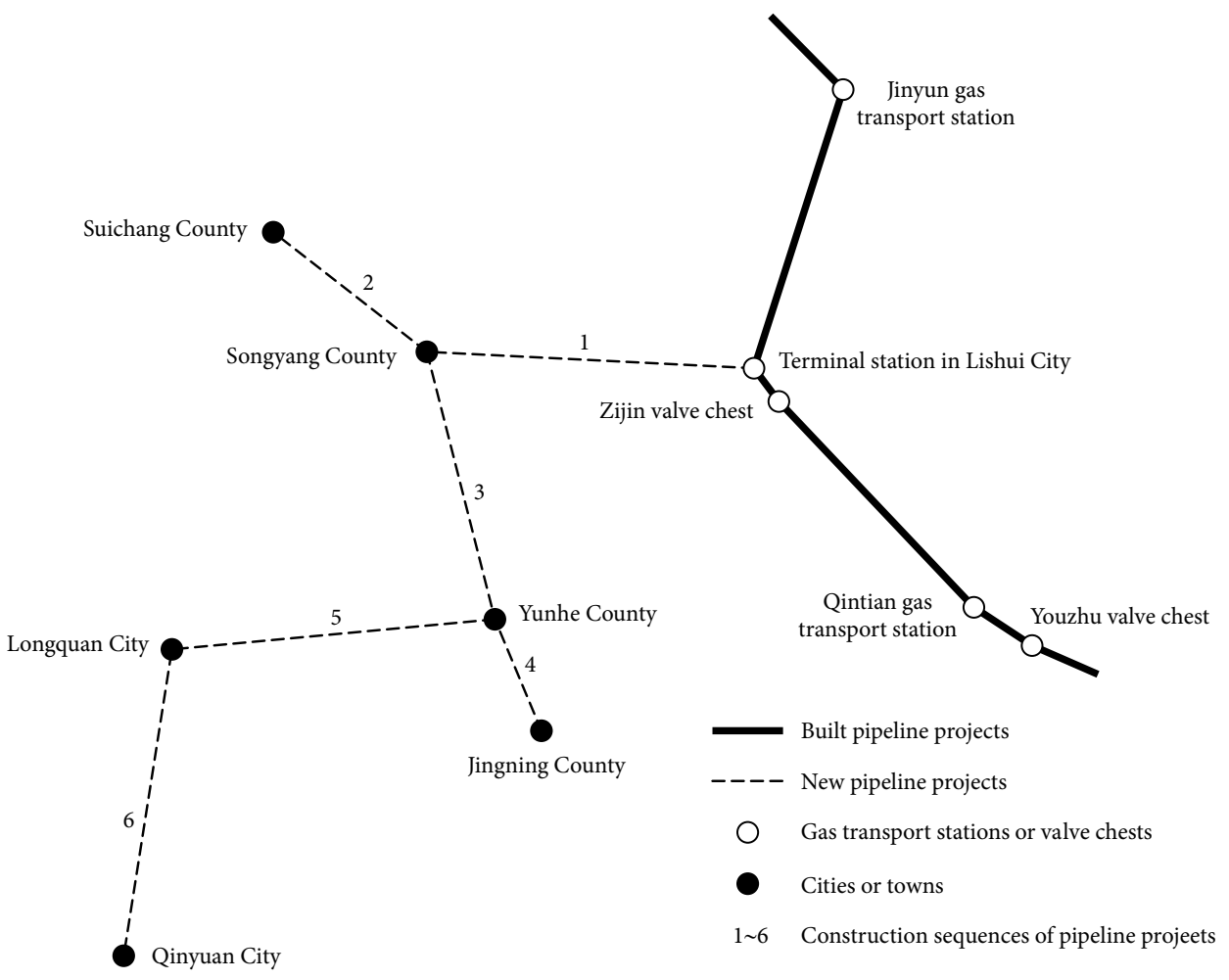

Fig. 4. Layout scheme of natural gas transport pipeline network 
Table 4. Model calculation results

\begin{tabular}{|c|l|l|c|}
\hline $\begin{array}{c}\text { Cycle } \\
\text { times }\end{array}$ & \multicolumn{1}{|c|}{$i$} & \multicolumn{1}{c|}{$j$} & $A_{i j}$ \\
\hline 1 & Songyang County & $\begin{array}{l}\text { Terminal station } \\
\text { in Lishui City }\end{array}$ & 6.63 \\
\hline 2 & Songyang County & Suichang & 18.85 \\
\hline 3 & Songyang County & Yunhe & 8.69 \\
\hline 4 & Yunhe County & Jingning & 28.52 \\
\hline 5 & Longquan City & Yunhe County & 6.14 \\
\hline 6 & Qinyuan County & Longquan City & 5.22 \\
\hline
\end{tabular}

\section{Conclusions}

The layout optimization of natural gas transport pipeline is a subject with social and economic benefits, but it is also a complex system problem. Based on influential factors analysis of natural gas transport pipeline projects' dominance degree, this paper proposes layout method of natural gas transport pipeline network based on DDM. The model can not only determine the optimal layout scheme, but also obtain construction sequences of gas transport pipeline projects, which ensure maximum comprehensive benefits of pipeline network. Natural gas transport pipeline projects in Zhejiang Province, China is taken as a case study, which suggest that the model should deal with the transport pipeline network layout problem well. The study finding has important implications for other potential pipeline networks not only in the Zhejiang Province but also throughout China and beyond.

With respect to calculating construction costs of pipeline projects, this paper mainly considers pipeline material consumption and costs of crossing obstacles, etc. However, during pipeline engineering design and construction process, construction costs of pipeline projects are also influenced by proportion of tunnels, topography and policy, etc. Therefore, how to quantify construction costs of natural gas transport pipeline projects comprehensively need to be further researched. In addition, the layout scheme of natural gas transport pipeline network can be obtained by DDM. However, distribution of natural gas, pipeline network technology and changes of airflow direction under different conditions should also be researched to determine the location and quantity of natural gas stations and valve chests reasonably and implement rational utilization of natural gas resources.

\section{Contribution}

Zhenjun Zhu and Chaoxu Sun conceived and designed the research; Zhenjun Zhu and Jun Zeng analyzed the data; Zhenjun Zhu wrote the paper; Chaoxu Sun and Guowei Chen helped improve the figures and manuscript.

All authors have read and approved the final manuscript.

\section{Disclosure Statement}

The authors declare that there are no conflict competing financial, professional and personal interests from other parties.

\section{References}

An, J.; Peng, S. 2016. Layout optimization of natural gas network planning: Synchronizing minimum risk loss with total cost, Journal of Natural Gas Science and Engineering 33: 255-263. https://doi.org/10.1016/j.jngse.2016.05.017

Bellman, R. E.; Dreyfus, S. E. 2016. Applied Dynamic Programming. Princeton University Press. 390 p.

Bhaskaran, S.; Salzborn, F. 1979. Optimal diameter assignment for gas pipeline networks, The Journal of the Australian Mathematical Society. Series B. Applied Mathematics 21(2):129-144.

https://doi.org/10.1017/S0334270000002009

Edgar, T. F.; Himmelblau, D. M.; Bickel, T. C. 1978. Optimal design of gas transmission networks, Society of Petroleum Engineers Journal 18(2). https://doi.org/10.2118/6034-PA

Chang, S. J. 2008. A cultural and philosophical perspective on Korea's education reform: a critical way to maintain Korea's economic momentum, Academic Paper Series 3(2): 1-11.

Graham, R. L.; Hell, P. 1985. On the history of the minimum spanning tree problem, Annals of the History of Computing 7(1): 43-57. https://doi.org/10.1109/MAHC.1985.10011

Gillingham, K.; Newell, R. G.; Palmer, K. 2009. Energy efficiency economics and policy, Annual Review of Resource Economics 1: 597-620.

https://doi.org/10.1146/annurev.resource.102308.124234

Han, D.-M.; Lim, J.-H. 2010. Design and implementation of smart home energy management systems based on ZigBee, IEEE Transactions on Consumer Electronics 56(3): 14171425. https://doi.org/10.1109/TCE.2010.5606278

Lu, H.; Taur, Y. 2006. An analytic potential model for symmetric and asymmetric DG MOSFETs, IEEE Transactions on Electron Devices 53(5): 1161-1168. https://doi.org/10.1109/TED.2006.872093

Mátyás, L. 1998. The gravity model: some econometric considerations, The World Economy 21(3): 397-401. https://doi.org/10.1111/1467-9701.00136

Novoselov, K. S.; Geim, A. K.; Morozov S. V.; Jiang, D.; Zhang, Y.; Dubonos, S. V.; Grigorieva, I. V.; Firsov, A. A. 2004. Electric field effect in atomically thin carbon films, Science. 306(5696): 666-669. https://doi.org/10.1126/science.1102896

Pedrycz, W.; Davidson, J.; Goulter, I. 1992. Neural network based decision model, used for design of rural natural gas systems, in IEEE International Conference on Fuzzy Systems1992, 8-12 March 1992, San Diego, CA, US, 12191226. https://doi.org/10.1109/FUZZY.1992.258651

Shamsie, J. 2003. The context of dominance: an industry-driven framework for exploiting reputation, Strategic Management Journal 24(3): 199-215. https://doi.org/10.1002/smj.291

Sniedovich, M. 2006. Dijkstra's algorithm revisited: the dynamic programming connexion, Control and Cybernetics 35(3): 599-620.

Sanaye, S; Mahmoudimehr, J. 2013. Optimal design of a natural gas transmission network layout, Chemical Engineering Research and Design 91(12): 2465-2476. https://doi.org/10.1016/j.cherd.2013.04.005 
Singh, R. R.; Nain, P. K. S. 2012. Optimization of natural gas pipeline design and its total cost using GA, International Journal of Scientific and Research Publications 2(8): 1-10.

Schmidt, M.; Steinbach, M. C.; Willert, B. M. 2015. High detail stationary optimization models for gas networks, Optimization and Engineering 16(1): 131-164.

https://doi.org/10.1007/s11081-014-9246-X

Üster, H.; Dilaveroğlu, S. 2014. Optimization for design and operation of natural gas transmission networks, Applied Energy 133: 56-59.

https://doi.org/10.1016/j.apenergy.2014.06.042

Zhou, H. 2004. Efficient Steiner tree construction based on spanning graphs, IEEE Transactions on Computer-Aided Design of Integrated Circuits and Systems 23(5): 704-710. https://doi.org/10.1109/TCAD.2004.826557 